\title{
Composición, abundancia y distribución de las poblaciones de gasterópodos de importancia comercial en La Guajira, Caribe colombiano
}

\author{
Ramón Nieto-Bernal ${ }^{1 *}$, Luis Chasqui V. ${ }^{1 *}$, Angélica María Rodríguez R. ${ }^{2}$, Erick Castro G. ${ }^{3}$ \& \\ Diego L. Gil-Agudelo ${ }^{1}$ \\ 1. Programa de Biodiversidad y Ecosistemas Marinos, Instituto de Investigaciones Marinas y Costeras, Cerro Punta de \\ Betín, Santa Marta, Colombia, A.A. 1016; ramonnieto2000@yahoo.es, luis_chasqui@invemar.org.co, \\ dl_gil@yahoo.com \\ 2. Programa de Maestría en Ciencias-Biología, Línea Biología Marina, Universidad Nacional de Colombia, Bogotá \\ D.C., Colombia; amrodriguezri@unal.edu.co \\ 3. Secretaria de Agricultura y Pesca, Gobernación Departamento Archipiélago de San Andrés, Providencia y Santa \\ Catalina, Colombia; ecastro@sanandres.gov.co \\ * Correspondencia
}

Recibido 30-I-2012. Corregido 15-IX-2012. Aceptado 11-X-2012.

\begin{abstract}
Composition, abundance and distribution of populations of commercially important gastropods in La Guajira, Colombian Caribbean. In the continental Colombian Caribbean the conch resource exploitation and the status of snails populations has been poorly studied, which are reflected in the lack of fisheries management. This study assesses composition, population density and distribution of the gastropods species that make conch resource in La Guajira region. Underwater visual censuses for snails were performed between September-November 2009 in $145100 \times 4 \mathrm{~m}\left(400 \mathrm{~m}^{2}\right)$ transects, spanning a total area of $56920 \mathrm{~m}^{2}$ between Riohacha and Cabo de la Vela. The study was complemented with the evaluation of composition, abundance and size of gastropods conch found in the discarded-by-fishermen shell mounds in 13 beaches. In October 2010 another 40 transects were evaluated $\left(16000 \mathrm{~m}^{2}\right)$ from the Southern of Riohacha to the Camarones village (La Guajira). We found a total of 9911 snails belonging to 12 species, the most abundant being Strombus pugilis with 8912 individuals and an average density of $1538.4 \pm 3662.6$ ind./ha, followed by Vasum muricatum with 374 individuals and an average density of 51.8 \pm 91.2 ind./ha. Calculating the importance value index (IVI) for both living organisms as the empty shells on beaches, shows that Turbinella angulata is the most used species by artisanal fishermen in the region. Cassis madagascariensis and Cassis tuberosa are also important snail resources in the region (as suggested by the number of empty shells found in beaches), but its densities were low. Strombus gigas, with only three living organisms found in the area, presented the lowest abundance ever found in the Colombian Caribbean ( $0.52 \pm 3.6$ ind./ha), showing that queen conch population in La Guajira cannot support commercial exploitation. The abundance of discarded $S$. gigas shells on beaches suggests resource exploitation in the recent past. Results remarks the urgency of implementing management plans for snail fisheries in the region. Rev. Biol. Trop. 61 (2): 683-700. Epub 2013 June 01.
\end{abstract}

Key words: gastropod, resource snail, Turbinella angulata, Strombus gigas, La Guajira, Colombian Caribbean.

Se estima que más del $75 \%$ de los recursos marinos del planeta se encuentran en estado de sobre-explotación (FAO 2005, Maguire et al. 2006). Un ejemplo de esto son los gasterópodos, que son utilizados principalmente como fuente de proteínas para humanos, además de ser usados mundialmente en la producción de artesanías, accesorios de moda como botones o aplicaciones en la formulación de lacas y champú (Nash 1993).

En todas las latitudes existen actividades pesqueras sobre el recurso caracol. En el Mar de Bering, por ejemplo, los caracoles de los géneros Neptunea y Buccinum soportan una alta presión pesquera desde principios de los 70's (MacIntosh 1980). En el Oeste del Océano 
Índico, Este del Pacífico y el Sudeste Asiático, el caracol verde (Turbo marmoratus), el caracol Trochus (Trochus niloticus) y los caracoles denominados abulones pertenecientes al género Haliotis tienen una alta demanda (Kitutani \& Yamakawa 1999); así como las especies Rapana venosa y $R$. thomassiana en el mar negro, y el bijarro común (Littorina sp.) en todo el Atlántico Norte (FAO 2005). En Chile y el sur de Perú, la especie Concholepas concholepas tiene una demanda apreciable por pescadores artesanales (Stotz 1997).

En el mar Caribe y las Antillas, los caracoles de mayor aprovechamiento han sido el caracol pala Strombus gigas (Theile 2002) y el burgao Cittarium pica (Schmidt et al. 2002, Robertson 2003). En el caso de S. gigas, la especie figura en el apéndice II de la CITES y por tanto su comercio es controlado internacionalmente (UNEP-WCMC 2011) debido a los críticos valores de abundancia poblacional en sus áreas de distribución (Berg \& Olsen 1989, Stoner \& Ray-Culp 2000, Gómez-Campo et al. 2010, Nieto-Bernal et al. 2011), al igual que para $C$. pica se han registrado críticos valores de abundancia poblacional en algunas regiones del Caribe, con densidades próximas a los 4.7ind. $/ \mathrm{m}^{2}$ (Osorno et al. 2009) las cuales son relacionadas con sobreexplotación (Leal 2002, Schmidt et al. 2002, Robertson 2003).

En algunas regiones del Caribe, la disminución de las poblaciones del caracol pala y el burgao, y las medidas de regulación adoptadas para su conservación, han conllevado el aumento de la explotación de otras especies de caracol como Turbinella angulata (Lightfoot 1786), Strombus costatus (Gmelin 1791) y Cassis madagascariensis (Lamarck, 1822), entre otros (Baqueiro 1997, Pérez-Pérez \& Aldana-Aranda 2000, FAO 2005, BaqueiroCárdenas 2004).

En el Caribe colombiano, la pesca del recurso caracol está compuesta principalmente por $S$. gigas, siendo permitida únicamente en algunos bancos del Archipiélago de San Andrés, Providencia y Santa Catalina, donde las poblaciones son evaluadas periódicamente y existe una cuota de pesca (Prada et al. 2008).
Le sigue en importancia Melongena melongena (Linnaeus, 1758), que a pesar de no existir información sobre su población en estado natural ni referencias biológico-pesqueras, es explotado de manera artesanal en casi todo el litoral del Caribe colombiano según información de la Corporación Colombia Internacional (CCI) y la oficina de pesca del Instituto Colombiano para el Desarrollo Rural (INCODER) (C. Borda, INCODER, Com. Per. 2010). C. pica es también aprovechado sin que existan estadísticas pesqueras sobre la explotación de este recurso (Osorno et al. 2009). Además, se conoce el comercio de conchas de algunas especies de la familia Strombidae (S. pugilis, Tricornis raninus y $S$. costatus), las pertenecientes al género Cassis y el caracol T. angulata, entre otras, en las tiendas de artesanías de las principales ciudades del Caribe colombiano (Poutiers \& Cipriani 1992, Obs. Pers. 2010), y de las cuales no existen estudios sobre sus poblaciones y estadísticas pesqueras.

En el pasado, la plataforma continental de La Guajira tuvo una importante pesca artesanal sobre el caracol pala $S$. gigas, aportando aproximadamente el 5\% de la producción total del recuso caracol en Colombia (Beltrán \& Villaneda 2000); sin embargo, ésta fue cerrada en el 2006 por sugerencia de la CITES debido a la falta de información sobre el estado de la población. No obstante, pescadores y residentes de la región afirman que el caracol pala junto con otras especies de gasterópodos son actualmente aprovechados, pero únicamente de manera artesanal.

Al presente, en la región de La Guajira no hay información sobre las especies de gasterópodos de interés comercial, además del estado y distribución actual de sus poblaciones. Por lo tanto, el objetivo del presente estudio fue evaluar la composición, densidad poblacional y distribución de las especies que conforman el recurso caracol en la región; además de un análisis de la presencia de conchas vacías en las playas, con el fin de describir las especies de caracol sobre las cuales se ha dirigido la presión pesquera en el área en los últimos años. La finalidad de este trabajo es contribuir 
con información básica para generar medidas de ordenamiento pesquero que promuevan la sustentabilidad del recurso caracol en la región.

\section{MATERIALES Y MÉTODOS}

Área de estudio: Los muestreos se realizaron entre septiembre-noviembre de 2009 en la región de La Guajira, desde Riohacha (11'34'49" N - 72॰54'33" W) hasta el Cabo de la Vela $\left(12^{\circ} 11^{\prime} 51^{\prime \prime} \mathrm{N}-72^{\circ} 10^{\prime} 0.3^{\prime \prime} \mathrm{W}\right)$ y en Octubre de 2010 desde el sur de Riohacha hasta

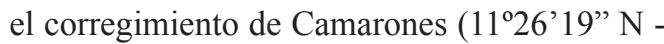
$73^{\circ} 04^{\prime} 50^{\prime \prime}$ W) (Fig. 1).

Abundancia, densidad y tallas: Mediante un mapa cartográfico de la zona de interés, se seleccionaron 184 estaciones entre los 3-20m de profundidad, separadas equidistantemente cada tres kilómetros. En cada estación se realizó un transecto de $400 \mathrm{~m}^{2}(100 \times 4 \mathrm{~m})$, donde se recolectaron y midieron ( $\mathrm{LT}=$ longitud total de la concha desde el ápice de la espira al extremo del canal sifonal) todos los caracoles de las diferentes especies que fueron identificados y liberados en la misma estación.

Preferencia de hábitat: Se realizó una descripción biofísica de cada estación valorando el porcentaje de cobertura de sustrato inerte (e.g. arena, cascajo) y organismos bentónicos sésiles (e.g. esponjas, corales duros, octocorales, pastos) mediante un cuadrante de $50 \times 50 \mathrm{~cm}$ al inicio, a los $25 \mathrm{~m}$ y $50 \mathrm{~m}$ del transecto, además de una estimación visual de la cobertura de cada sustrato a través de todo el transecto. En cada estación, se tomó una muestra de $300 \mathrm{~g}$ de sedimento para realizar un análisis granulométrico y de materia orgánica. Los valores de granulometría por estación fueron expresados en un solo valor que corresponde al tamaño medio del grano del sedimento (Folk 1980). El contenido de materia orgánica (MO) fue medido por la técnica de pérdida de peso por

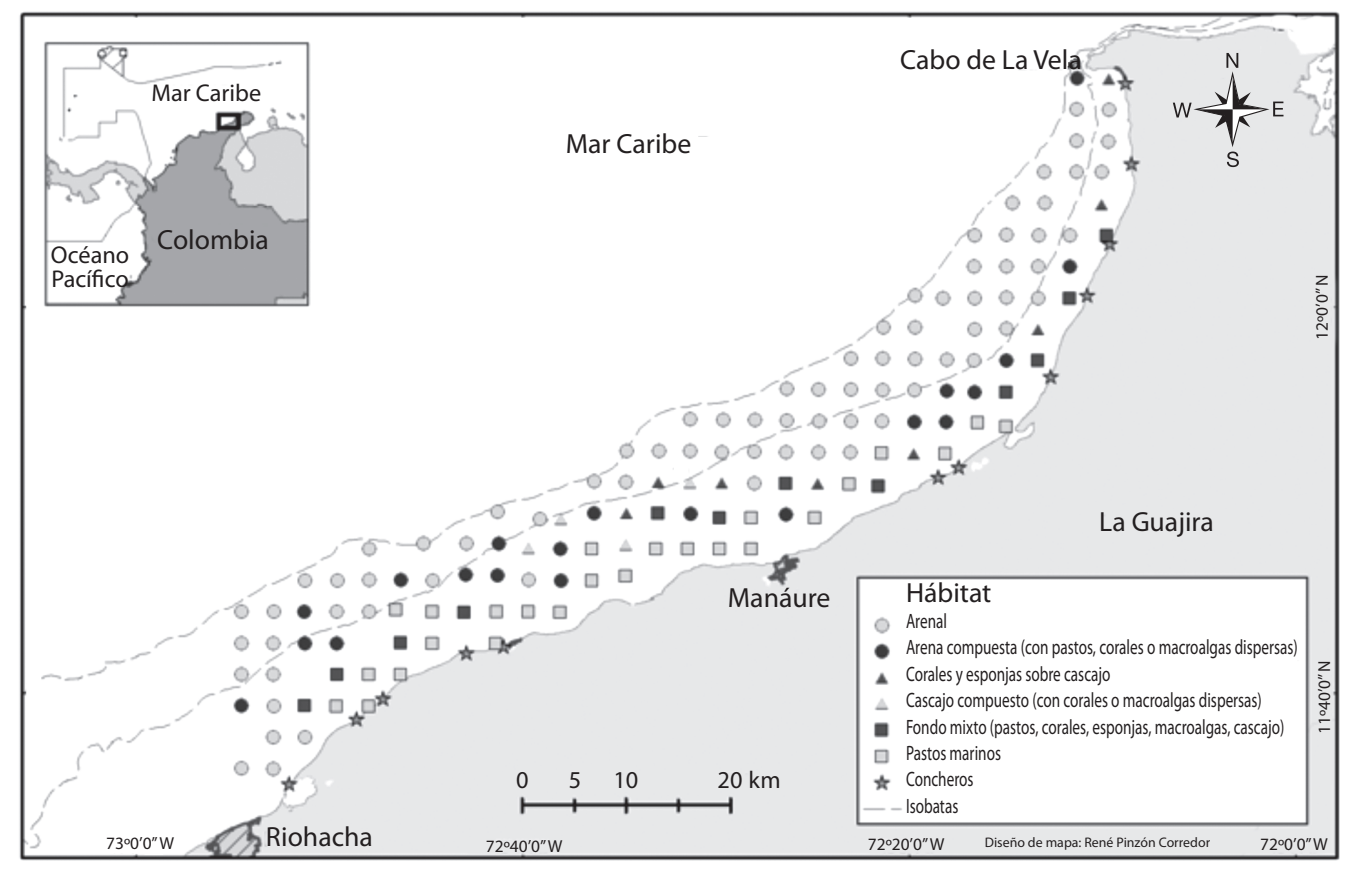

Fig. 1. Hábitats encontrados en la plataforma continental de La Guajira durante la evaluación de 2009 desde Riohacha hasta Cabo de la Vela, entre 3-20m de profundidad y "concheros" visitados en las playas.

Fig. 1. Habitats found on La Guajira continental shelf in the 2009 assessment from Riohacha to Cabo de la Vela 3-20m depth and "concheros" visited on the beaches. 
ignición de cinco gramos de sedimento a $600^{\circ} \mathrm{C}$ por dos horas en una mufla (Buchanan 1984, Luczak et al. 1997). La diferencia de peso entre la muestra seca y la muestra calcinada corresponde a la materia orgánica, la que fue expresada como porcentaje del peso seco.

Importancia comercial: Para conocer las principales especies capturadas por los pescadores locales, se efectuó una evaluación de los botaderos de conchas de caracol con señales de pesca (concheros) encontrados en 13 playas a lo largo del litoral entre Cabo de la Vela y Riohacha (Fig. 1). En cada playa se evalúo un área aproximada de $2000 \mathrm{~m}^{2}$ donde se registró el número de conchas por especie y la longitud total de cada una. La evaluación de los botaderos de conchas en playas es un método que contribuye a reflejar cómo fue en el pasado la presión pesquera sobre el recurso caracol (Stoner \& Ray 1996, Stoner 1997, Torres \& Sullivan-Sealey 2002a, Schapira et al. 2009).

Análisis de los datos: De acuerdo a la descripción biofísica, a cada estación se le asignó una categoría en relación con el tipo de hábitat encontrado conforme a las coberturas del sustrato (Cuadro 1).

Se determinó para cada especie su abundancia total (abundancia absoluta) y su porcentaje respecto al total de individuos de todas las especies (abundancia relativa), frecuencia de ocurrencia absoluta (número de estaciones en que se observó la especie), frecuencia de ocurrencia relativa (porcentaje de estaciones en que se observó con respecto al total de estaciones evaluadas) y densidad poblacional en la totalidad del área muestreada y en cada categoría de hábitat encontrado, expresada en el número de individuos por hectárea (ind./ ha). Se calculó el índice de valor de importancia (IVI=abundancia relativa + frecuencia relativa/2; en porcentaje) para cada especie (Lamprecht 1990). Este índice es una medida de cuantificación que asigna a cada especie su categoría de importancia, expresando el predominio de cada especie dentro del grupo de organismos al considerar la abundancia y la frecuencia relativa (Brower et al. 1998). Este índice también se utilizó para los datos obtenidos en la evaluación de los botaderos de conchas en las playas.

Mediante tablas de contingencia y correlaciones de Spearman se evalúo la relación entre la abundancia de las especies con la profundidad, el contenido de materia orgánica del sedimento, el tamaño medio de grano del sedimento y el porcentaje de cobertura del tipo de sustrato (arena, cascajo, gorgonáceos, coral duro, esponjas, pastos marinos, macroalgas). Para las especies más abundantes se estimó el índice de preferencia por hábitat, definido por la diferencia entre el porcentaje de individuos encontrado en cada hábitat y el porcentaje del área total que este ocupa (Appeldoorn \& Rolke 1996). Un valor positivo del índice muestra la

CUADRO 1

Hábitats encontrados en la plataforma continental de La Guajira (Colombia) desde Riohacha hasta Cabo de la Vela entre 3-20m de profundidad en 2009

TABLE 1

Habitats found on La Guajira continental shelf (Colombia) from Riohacha to Cabo de la Vela between 3-20m depth in 2009

\begin{tabular}{llc}
\multicolumn{1}{c}{ Tipo de hábitat } & \multicolumn{1}{c}{ Descripción (\% cobertura sustrato) } & Código \\
Plano de arena & Arena $\geq 90 \%$ & Are \\
Arena compuesta (con pastos, corales o macroalgas dispersas) & Arena entre $60 \%$ y $90 \%$ & ACm \\
Cascajo compuesto (con corales o macroalgas dispersas) & Cascajo entre $60 \%$ y $90 \%$ & CCm \\
Pradera de pastos (Thalassia testudinum y Syringodium filiforme) & Pastos $>70 \%$ & Pas \\
Fondo mixto (pastos, corales, esponjas, macroalgas, cascajo) & Sustratos entre $30 \%$ y $60 \%$ & FMx \\
Gorgonáceos y esponjas sobre cascajo & Gorgonáceos y Esponjas $\geq 70 \%$ & GEC \\
\hline
\end{tabular}


afinidad de la especie a determinado hábitat teniendo en cuenta la proporción disponible del mismo, un valor negativo indica lo contrario (Appeldoorn \& Rolke 1996, Schweizer \& Posada 2006). Adicionalmente, para la especie con mayor frecuencia de aparición se evaluó la relación entre la abundancia (variable respuesta) y las variables ambientales mencionadas en conjunto (variables explicativas), mediante un modelo aditivo generalizado (GAM, por su siglas en inglés) (Hastie \& Tibshirani 1990, Crawley 2007).

\section{RESULTADOS}

Abundancia, densidad y tallas: Durante los muestreos realizados en 2009 se evaluó un área de $56920 \mathrm{~m}^{2}$, donde se encontraron 9602 caracoles pertenecientes a 12 especies (Cuadro 2). Strombus pugilis fue la especie más abundante con un IVI de $67.5 \%$, presentando una talla promedio de $68.1 \pm 25.4 \mathrm{~mm}$ LT y una densidad promedio de $1538.4 \pm 3662.6$ ind./ ha. La especie más frecuente fue $T$. angulata, con un valor de IVI de $31.4 \%$, una longitud promedio de $173.1 \pm 25.4 \mathrm{~mm}$ LT y densidad de $34.5 \pm 42.1 \mathrm{ind}$./ha. Las especies que presentaron los valores de IVI más bajos fueron Cymatium femorale y Cassis tuberosa, ambos con el $0.7 \%$, con tallas promedio de $116.3 \pm 10.5 \mathrm{~mm}$ y $223.0 \pm 51.1 \mathrm{~mm}$, respectivamente. $S$. gigas tuvo un IVI de $1.1 \%$, encontrándose solo dos individuos adultos (Longitud del labio $>5 \mathrm{~mm}$ ) con tallas de 208 y $285 \mathrm{~mm}$ LT, y un individuo inmaduro (Longitud del labio $<5 \mathrm{~mm}$ ) con una talla de $237 \mathrm{~mm}$ LT.

En 2010 se evaluó un área de $15600 \mathrm{~m}^{2}$, encontrándose un total de 309 caracoles pertenecientes a nueve especies (Cuadro 3). T. angulata presentó un valor de IVI de $33.7 \%$, siendo nuevamente la especie más frecuente, con una talla promedio de $199.94 \pm 54.0 \mathrm{~mm}$ LT y una densidad de $43.5 \pm 39.6$ ind./ha, seguido de $V$. muricatum con un IVI de $33.5 \%$, con una talla promedio de $75.26 \pm 52.4 \mathrm{~mm}$ LT y densidad de $61.3 \pm 149.0$ ind./ha. Igualmente, como en el muestreo anterior, C. tuberosa fue la especie con el valor de IVI más bajo (1.4\%), presentando un solo ejemplar. Durante este muestreo

CUADRO 2

Índice de valor de importancia (IVI= abundancia relativa + frecuencia relativa / 2; en porcentaje), densidad y longitud total (LT) de las especies de gasterópodos encontrados en La Guajira (Colombia) desde Riohacha hasta Cabo de la Vela entre 3-20m de profundidad en 2009

TABLE 2

Importance value index (IVI $=$ relative abundance + relative frequency $/ 2$ in percentage), density and total length (TL) of the gastropod species found in La Guajira (Colombia) from Riohacha to Cabo de la Vela between 3-20m depth in 2009

\begin{tabular}{lcccccccc}
\multicolumn{1}{c}{ Especie } & IVI (\%) & $\begin{array}{c}\text { Abundancia } \\
\text { absoluta (n) }\end{array}$ & $\begin{array}{c}\text { Abundancia } \\
\text { relativa (\%) }\end{array}$ & $\begin{array}{c}\text { Frecuencia } \\
\text { absoluta }\end{array}$ & $\begin{array}{c}\text { Frecuencia } \\
\text { relativa (\%) }\end{array}$ & $\begin{array}{c}\text { Densidad promedio } \\
\text { (ind./ha) }\end{array}$ & $\begin{array}{c}\text { LT promedio } \\
(\mathrm{mm})\end{array}$ \\
Strombus pugilis & 67.48 & 8846 & 92.19 & 62 & 42.76 & $1538.4 \pm 3662.6$ & $68.1 \pm 25.4$ \\
Turbinella angulata & 31.39 & 200 & 2.08 & 88 & 60.69 & $34.5 \pm 42.1$ & $173.1 \pm 25.4$ \\
Vasum muricatum & 21.90 & 298 & 3.11 & 59 & 40.69 & $51.8 \pm 91.2$ & $71.8 \pm 25.2$ \\
Phyllonotus margaritensis & 14.42 & 121 & 1.26 & 40 & 27.59 & $20.6 \pm 58.6$ & $91.6 \pm 23.9$ \\
Tricornis raninus & 10.98 & 55 & 0.57 & 31 & 21.38 & $9.5 \pm 22.5$ & $90.1 \pm 25.0$ \\
Chicoreus brevifrons & 6.76 & 40 & 0.42 & 19 & 13.10 & $6.9 \pm 28.3$ & $101.7 \pm 24.1$ \\
Cymatium parthenopeum & 1.46 & 16 & 0.17 & 4 & 2.76 & $2.8 \pm 20.6$ & $93.4 \pm 17.2$ \\
Cassis madagascariensis & 1.76 & 6 & 0.06 & 5 & 3.45 & $1.03 \pm 5.8$ & $189.3 \pm 22.9$ \\
Fasciolaria tulipa & 1.75 & 5 & 0.05 & 5 & 3.45 & $0.86 \pm 4.6$ & $114.8 \pm 23.8$ \\
Strombus gigas & 1.05 & 3 & 0.03 & 3 & 2.07 & $0.52 \pm 3.6$ & $243.3 \pm 26.1$ \\
Cymatium femorale & 0.71 & 3 & 0.03 & 2 & 1.38 & $0.52 \pm 4.6$ & $116.3 \pm 10.5$ \\
Cassis tuberosa & 0.70 & 2 & 0.02 & 2 & 1.38 & $0.34 \pm 2.9$ & $223.0 \pm 51.1$ \\
\hline
\end{tabular}


CUADRO 3

Índice de valor de importancia (IVI= abundancia relativa + frecuencia relativa / 2 ; en porcentaje), densidad y longitud total (LT) de las especies de gasterópodos encontrados en La Guajira (Colombia) desde Riohacha hasta corregimiento de Camarones (La Guajira) entre 3-20m de profundidad en 2010

TABLE 3

Importance value index (IVI = relative abundance + relative frequency / 2 in percentage), density and total length (TL) of the gastropod species found in La Guajira (Colombia) from Riohacha to the Camarones village (La Guajira) between 3-20m depth in 2010

\begin{tabular}{lccccccc}
\multicolumn{1}{c}{ Especie } & $\begin{array}{c}\text { IVI } \\
(\%)\end{array}$ & $\begin{array}{c}\text { Abundancia } \\
\text { absoluta (n) }\end{array}$ & $\begin{array}{c}\text { Abundancia } \\
\text { relativa (\%) }\end{array}$ & $\begin{array}{c}\text { Frecuencia } \\
\text { absoluta }\end{array}$ & $\begin{array}{c}\text { Frecuencia } \\
\text { relativa (\%) }\end{array}$ & $\begin{array}{c}\text { Densidad total } \\
\text { (ind./ha) }\end{array}$ & $\begin{array}{c}\text { LT promedio } \\
(\mathrm{mm})\end{array}$ \\
Turbinella angulata & 33.74 & 54 & 17.48 & 20 & 50.0 & $43.5 \pm 39.6$ & $199.94 \pm 54.0$ \\
Vasum muricatum & 33.55 & 76 & 24.60 & 17 & 42.5 & $61.3 \pm 149.0$ & $75.26 \pm 52.4$ \\
Tricornis raninus & 24.47 & 74 & 23.95 & 10 & 25.0 & $59.7 \pm 176.4$ & $78.91 \pm 48.5$ \\
Strombus pugilis & 16.93 & 66 & 21.36 & 5 & 12.5 & $53.2 \pm 190.6$ & $68.92 \pm 57.43$ \\
Chicoreus brevifrons & 9.81 & 22 & 7.12 & 5 & 12.5 & $17.7 \pm 75.5$ & $81.32 \pm 57.46$ \\
Phyllonotus margaritensis & 9.60 & 13 & 4.21 & 6 & 15.0 & $10.5 \pm 25.2$ & $85.84 \pm 47.29$ \\
Cassis madagascariensis & 2.82 & 2 & 0.65 & 2 & 5.0 & $1.6 \pm 6.1$ & $176 \pm 52.7$ \\
Cassis flamea & 1.41 & 1 & 0.32 & 1 & 2.5 & $0.8 \pm 4.4$ & 184 \\
Cassis tuberosa & 1.41 & 1 & 0.32 & 1 & 2.5 & $0.8 \pm 4.4$ & 210 \\
\hline
\end{tabular}

no se registraron individuos pertenecientes a la especie $S$. gigas.

Preferencia de hábitat: Según la composición del sustrato las estaciones se agruparon en seis diferentes hábitats (Cuadro 1). El hábitat más común fue el conformado por planos arenosos (Are) cubriendo el 51.6\% del área total muestreada; seguido por el de pastos marinos (Pas, compuesto principalmente por Thalassia testudinum) con un área de $17.4 \%$, mientras que los hábitats de Gorgonáceos y esponjas sobre cascajo (GEC) y los de Fondo mixto (FMx) representaron un $5.6 \%$ y $9.2 \%$ respectivamente (Fig. 2).

De acuerdo al análisis de tablas de contingencia (Cuadro 4), se observaron asociaciones de las especies de caracoles con algunos tipos de sustrato ( $\mathrm{V}$ de Cramér $=0.35$, M-L $\left.\mathrm{X}^{2}=2796.73, \mathrm{p}<0.01\right)$. La abundancia del caracol S. pugilis se correlacionó positivamente con el sustrato arenoso $\left(\%\right.$ Arena $\left.\mathrm{r}_{\mathrm{s}}=0.559, \mathrm{p}<0.05\right)$. Además, según el gráfico de distribución de las especies por tipos de hábitats (Fig. 2) y el índice de preferencia de hábitat (IP) (Cuadro 5), $S$. pugilis fue más representativo en los ambientes con amplias coberturas de arena y cascajo.
T. angulata fue más abundante en los sustratos conformados por cascajo, gorgonáceos y esponjas $\left(\mathrm{r}_{\mathrm{s}}, \mathrm{p}<0.05\right)$, presentando una correlación negativa con sustratos compuestos por pastos marinos (\% Pastos $r_{\mathrm{s}}=-0.403$, $\mathrm{p}<0.05)$. El modelo de distribución GAM, fue realizado solamente para $T$. angulata por ser la especie con mayor frecuencia de aparición, además siendo el único modelo que presentó una variabilidad en la distribución de los datos mayor al 30\%. Se encontró que la abundancia de $T$. angulata está siendo explicada significativamente $(\mathrm{p}<0.01)$ por la profundidad y el porcentaje de materia orgánica del sedimento (\%MO), y que el $61.1 \%$ de su variabilidad es explicada por el modelo (Cuadro 6). Sin embargo, al observar las gráficas que exponen estas relaciones, se encontró que para la profundidad son poco notorios los cambios de abundancia de T. angulata (Fig. 3). No obstante, las mayores abundancias se dieron entre $8-13 \mathrm{~m}$. Igualmente sucedió con $\% \mathrm{MO}$, encontrándose cambios no muy evidentes con relación a estos valores (Fig. 3). Aun así, puede visualizarse que entre $3-5 \%$ de $\mathrm{MO}$ se presentan las mayores abundancias de este caracol. 

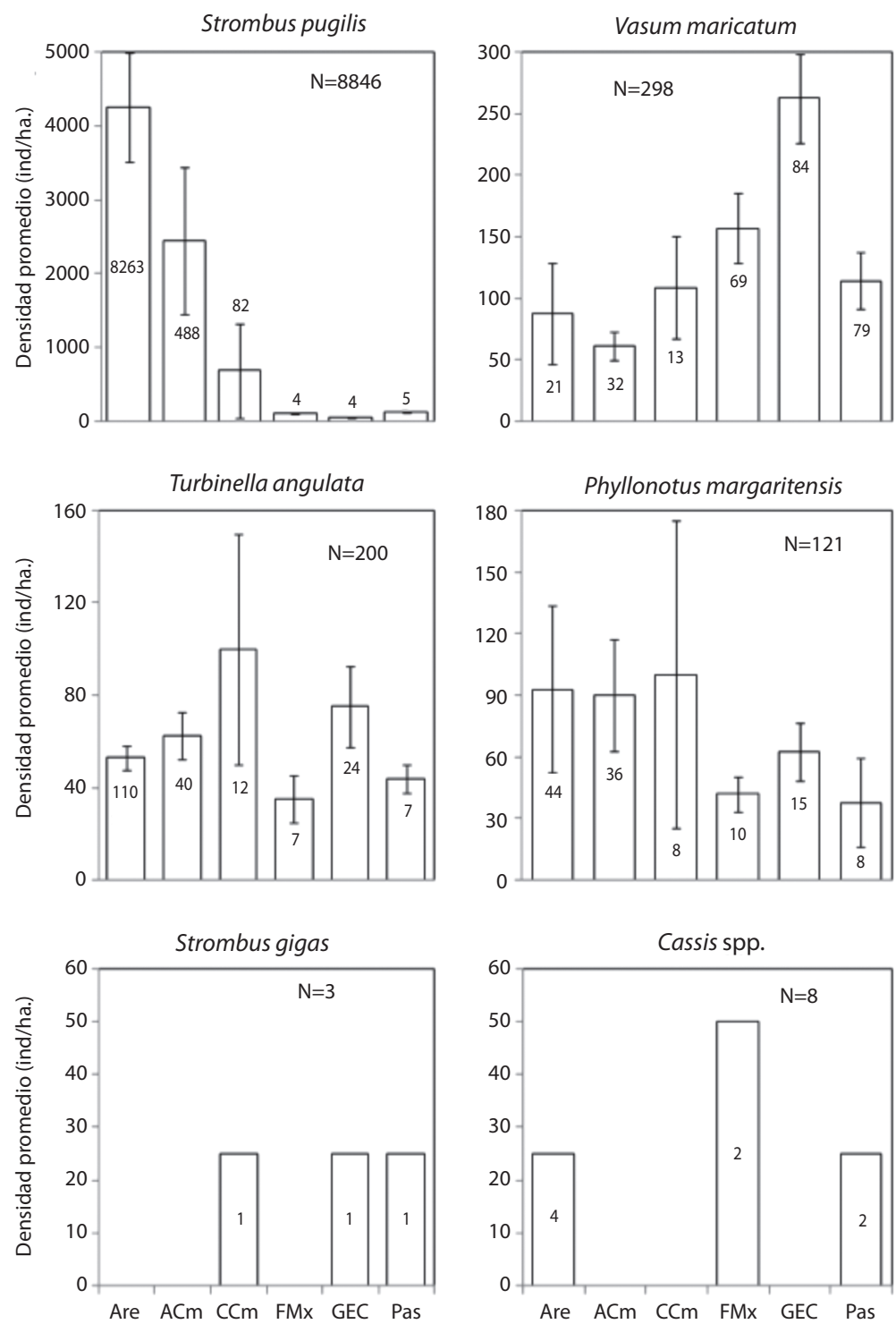

Fig. 2. Densidad promedio de los gasterópodos más abundantes y los de interés comercial por tipo de ambiente en la plataforma continental de La Guajira (Colombia) desde Riohacha hasta Cabo de la Vela entre 3-20m de profundidad. Ver cuadro 2 para los códigos de los tipos de hábitat.

Fig. 2. Average density of the most abundant and commercially important gastropod species by habitat type on the La Guajira continental shelf (Colombia) from Riohacha to Cabo de la Vela 3-20m depth. See table 2 for habitat codes.

Por otro lado, $V$. muricatum tuvo sus mayores densidades en los hábitats de Gorgonáceos y esponjas sobre cascajo, Fondo mixto y Cascajo compuesto ( $\%$ Cascajo $\mathrm{r}_{\mathrm{s}}=0.479, \%$ Gorgonáceos $\mathrm{r}_{\mathrm{s}}=0.422, \mathrm{p}<0.05$; Cuadro 5, Fig.
2). Los dos ejemplares adultos de $S$. gigas se hallaron en los hábitats de Cascajo compuesto, Gorgonáceos y esponjas sobre cascajo y el ejemplar inmaduro sobre Praderas de pastos (Fig. 2). De los ocho individuos pertenecientes 
CUADRO 4

Frecuencias de encuentro de las especies de caracol en cada tipo de hábitat en la plataforma continental de La Guajira. Ver cuadro 1 para la descripción de los tipos de hábitats

TABLE 4

Frequency sighting of the snail species in each habitat type in the continental shelf of La Guajira. See Table 1 for descriptions of habitat types

\begin{tabular}{|c|c|c|c|c|c|c|c|}
\hline \multirow{2}{*}{ Especie } & \multicolumn{6}{|c|}{ Tipo de Hábitat } & \multirow{2}{*}{ Total de la fila } \\
\hline & Are & $\mathrm{ACm}$ & FMx & Pas & $\mathrm{CCm}$ & GEC & \\
\hline \multirow[t]{2}{*}{ Strombus pugilis } & 8263 & 488 & 4 & 5 & 82 & 4 & 8846 \\
\hline & 86.12 & 5.09 & 0.04 & 0.05 & 0.85 & 0.04 & 92.19 \\
\hline \multirow[t]{2}{*}{ Turbinella angulata } & 110 & 40 & 7 & 7 & 12 & 24 & 200 \\
\hline & 1.15 & 0.42 & 0.07 & 0.07 & 0.13 & 0.25 & 2.08 \\
\hline \multirow[t]{2}{*}{ Vasum muricatum } & 21 & 32 & 69 & 79 & 13 & 84 & 298 \\
\hline & 0.22 & 0.33 & 0.72 & 0.82 & 0.14 & 0.88 & 3.11 \\
\hline \multirow[t]{2}{*}{ Tricornis raninus } & 11 & 15 & 5 & 12 & 5 & 7 & 55 \\
\hline & 0.11 & 0.16 & 0.05 & 0.13 & 0.05 & 0.07 & 0.57 \\
\hline \multirow[t]{2}{*}{ Cassis madagascariensis } & 3 & 0 & 2 & 1 & 0 & 0 & 6 \\
\hline & 0.03 & 0 & 0.02 & 0.01 & 0 & 0 & 0.06 \\
\hline \multirow[t]{2}{*}{ Phyllonotus margaritensis } & 44 & 36 & 10 & 8 & 8 & 15 & 121 \\
\hline & 0.46 & 0.38 & 0.1 & 0.08 & 0.08 & 0.16 & 1.26 \\
\hline \multirow[t]{2}{*}{ Cymatium parthenopeum } & 1 & 0 & 0 & 7 & 0 & 8 & 16 \\
\hline & 0.01 & 0 & 0 & 0.07 & 0 & 0.08 & 0.17 \\
\hline \multirow[t]{2}{*}{ Fasciolaria tulipa } & 0 & 0 & 1 & 3 & 0 & 1 & 5 \\
\hline & 0 & 0 & 0.01 & 0.03 & 0 & 0.01 & 0.05 \\
\hline \multirow[t]{2}{*}{ Chicoreus brevifrons } & 7 & 4 & 6 & 1 & 9 & 13 & 40 \\
\hline & 0.07 & 0.04 & 0.06 & 0.01 & 0.09 & 0.14 & 0.42 \\
\hline \multirow[t]{2}{*}{ Cassis tuberosa } & 1 & 0 & 0 & 1 & 0 & 0 & 2 \\
\hline & 0.01 & 0 & 0 & 0.01 & 0 & 0 & 0.02 \\
\hline \multirow[t]{2}{*}{ Strombus gigas } & 0 & 0 & 0 & 1 & 1 & 1 & 3 \\
\hline & 0 & 0 & 0 & 0.01 & 0.01 & 0.01 & 0.03 \\
\hline \multirow[t]{2}{*}{ Cymatium femorale } & 1 & 0 & 2 & 0 & 0 & 0 & 3 \\
\hline & 0.01 & 0 & 0.02 & 0 & 0 & 0 & 0.03 \\
\hline \multirow[t]{2}{*}{ Total de la columna } & 8462 & 615 & 106 & 125 & 130 & 157 & 9595 \\
\hline & 88.19 & 6.41 & 1.1 & 1.3 & 1.35 & 1.64 & 100 \\
\hline
\end{tabular}

al género Cassis, se encontraron cuatro en los hábitats de Plano de arena y dos en los de Fondos mixtos y Pastos marinos, respectivamente.

Importancia comercial: En las 13 playas evaluadas se encontró un total de 430 conchas de caracol pertenecientes a cuatro especies de importancia comercial en la región (Cuadro 7). La concha de $T$. angulata tuvo el mayor valor de importancia (IVI=67.3\%), se encontró en 12 de los 13 concheros evaluados, y presentó una talla promedio de $206.9 \pm 52.9 \mathrm{~mm}$
LT. Le siguieron $S$. gigas con un IVI de $32.7 \%$ y una talla promedio de $245.7 \pm 40.9 \mathrm{~mm}$ LT y C. madagascariensis con un IVI de $22.1 \%$ y una talla promedio de $189.6 \pm 25.3 \mathrm{~mm}$ LT (Cuadro 7).

\section{DISCUSIÓN}

Abundancia, densidad, tallas y preferencia de hábitat: La plataforma continental de la península de La Guajira, es una extensa región del Caribe colombiano con una 


\section{CUADRO 5}

Índice de preferencia de hábitats de las especies de gasterópodos más abundantes en la plataforma continental de

La Guajira (Colombia) desde Riohacha hasta Cabo de la Vela entre 3-20m de profundidad. IP =\% de individuos por especie - \% área. Para este índice un valor positivo indica la afinidad de la especie al tipo de hábitat.

(Descripción del hábitat: ver cuadro 1; Especies: Spug = Strombus pugilis. Vmur = Vasum muricatum .

Tang = Turbinella angulata . Pmar = Phyllonotus margaritensis $)$

TABLE 5

Habitat preference index of the most abundant gastropod species on La Guajira continental shelf (Colombia) from Riohacha to Cabo de la Vela 3-20m depth. IP = individuals by specie (\%) - area (\%). Positive values indicates species affinity to habitat type. (Description of habitat: see table 1; Species: Spug = Strombus pugilis. Vmur $=$ Vasum muricatum. Tang $=$ Turbinella angulata. Pmar $=$ Phyllonotus margaritensis $)$

\begin{tabular}{lcccccccccc}
\multicolumn{1}{c}{ Hábitat } & \multicolumn{3}{c}{ Porcentaje (\%) } & \multicolumn{5}{c}{ Índice de preferencia (IP) } \\
& Área & Spug & Vmur & Tang & Pmar & Spug & Vmur & Tang & Pmar \\
Are & 51.6 & 97.6 & 0.2 & 1.3 & 0.5 & 46 & -51.4 & -50.3 & -51.1 \\
ACm & 13.4 & 79.3 & 5.2 & 6.5 & 5.9 & 66 & -8.2 & -6.9 & -7.5 \\
CCm & 2.8 & 63.1 & 10 & 9.2 & 6.2 & 60.3 & 7.2 & 6.4 & 3.3 \\
GEC & 5.6 & 2.5 & 53.5 & 15.3 & 9.6 & -3.1 & 47.9 & 9.7 & 3.9 \\
FMx & 9.2 & 3.8 & 65.1 & 6.6 & 9.4 & -5.4 & 55.9 & -2.5 & 0.3 \\
Pas & 17.4 & 4 & 63.2 & 5.6 & 6.4 & -13.4 & 45.8 & -11.8 & -11 \\
\hline
\end{tabular}

\section{CUADRO 6}

Modelo aditivo generalizado (GAM, siglas del nombre en inglés) para la relación entre la abundancia de Turbinella angulata y las variables ambientales, encontrada en La Guajira (Colombia) desde Riohacha hasta Cabo de la Vela entre 3-20m de profundidad en 2009. e.e: error estándar. Nivel de significancia: $0.01 *$

TABLE 6

Generalized additive model (GAM) for the relationship between the abundance of Turbinella angulata and the environmental variables, found in La Guajira (Colombia) from Riohacha to Cabo de la Vela 3-20m depth in 2009. e.e: standard error. Level of significance: $0.01 *$

\begin{tabular}{lc}
\multicolumn{1}{c}{ Variables explicativas } & Varible respuesta: Abudancia T. angulata \\
Profundidad & $0.0466 *$ \\
$\%$ Materia orgánica sedimento & $0.0134 *$ \\
Familia de distribución & Poisson \\
Función de enlace & Logaritmo \\
Variabilidad explicada (\%) & $61.1 \%$ \\
Coef. determinación $\left(\mathrm{r}^{2}\right)$ & 0.502 \\
Intercepto (e.e) & $-7.273(6.980)$ \\
$\mathrm{N}$ & 145 \\
\hline
\end{tabular}

alta productividad biológica (Suzunaga et al. 1992), donde el componente bentónico es el predominante dentro de la red trófica (CrialesHernández et al. 2006), el cual se dispersa, según sus requerimientos, sobre un mosaico ambiental conformado por distintos tipos de sustrato (Díaz-Pulido 2000, Gómez-López 2009, Nieto-Bernal et al. 2011). Esto se refleja en el comportamiento de las poblaciones de gasterópodos de interés comercial en esta zona del Caribe.

En el caso de $S$. pugilis, se encontraron grandes agregaciones (hasta de 21775 ind./ ha en una estación) sobre extensos fondos arenosos entre los 5-20m de profundidad, muy distinto a lo visto en las praderas de pastos marinos donde la densidad fue comparativamente menor. En las zonas centro y norte del 

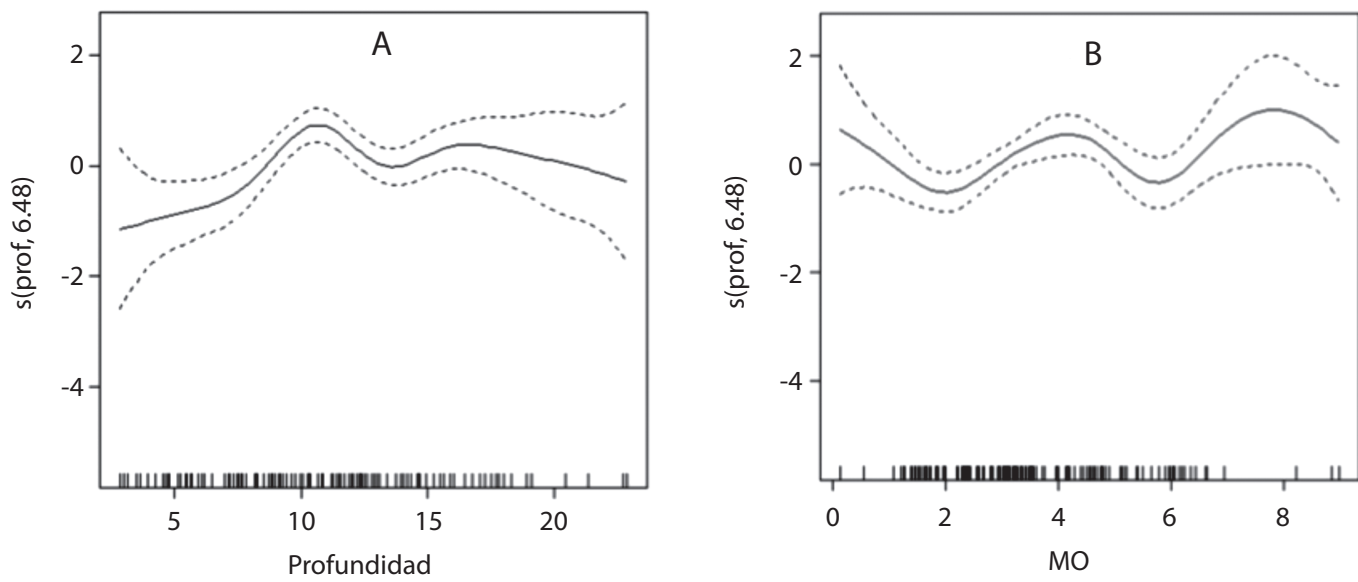

Fig. 3. Modelo aditivo generalizado (GAM, siglas del nombre en inglés) para la relación entre la abundancia de Turbinella angulata y las variables ambientales: A) profundidad y B) porcentaje de materia orgánica del sedimento (\%MO), encontrada en la plataforma continental de La Guajira (Colombia) desde Riohacha hasta Cabo de la Vela, entre 3-20m de profundidad. En el eje Y los residuales parciales de la abundancia del caracol.

Fig. 3. Plot of the generalized additive model (GAM) for the relationship between the abundance of Turbinella angulata and environmental variables: A) depth and B) percentage of sediment organic matter (\%MO), found in La Guajira continental shelf (Colombia) from Riohacha to Cabo de la Vela, between 3-20m depth. On the Y axis the partial residual abundance of the conch.

\section{CUADRO 7}

Información general sobre las conchas de caracol encontradas en las playas de La Guajira (Colombia) desde Riohacha hasta Cabo de la Vela. Abundancia (N) y longitud total (LT)

TABLE 7

General information about conchs found on La Guajira beaches (Colombia) from Riohacha to Cabo de la Vela. Abundance $(\mathrm{N})$ and total length (LT)

\begin{tabular}{lcccccc}
\multicolumn{1}{c}{ Especie } & $\begin{array}{c}\text { Abundancia } \\
\text { absoluta (n) }\end{array}$ & $\begin{array}{c}\text { Abundancia } \\
\text { relativa (\%) }\end{array}$ & $\begin{array}{c}\text { Frecuencia } \\
\text { absoluta }\end{array}$ & $\begin{array}{c}\text { Frecuencia } \\
\text { relativa (\%) }\end{array}$ & IVI (\%) & $\begin{array}{c}\text { LT Promedio } \\
(\mathrm{mm})\end{array}$ \\
Turbinella angulata & 218 & 42.25 & 12 & 92.31 & 67.28 & $206.99 \pm 52.94$ \\
Strombus gigas & 99 & 19.19 & 6 & 46.15 & 32.67 & $245.68 \pm 40.88$ \\
Cassis madagascariensis & 69 & 13.37 & 4 & 30.77 & 22.07 & $189.58 \pm 25.25$ \\
Cassis tuberosa & 44 & 8.53 & 2 & 15.38 & 11.96 & $193.48 \pm 21.9$ \\
\hline
\end{tabular}

Gran Caribe se ha reportado que los adultos de $S$. pugilis tienden a formar agregaciones de cientos de individuos en fondos arenosos entre los 3-30m de profundidad durante todo el año (Percharde 1982, Catterall \& Poiner 1983, Sanders 1988, Reed 1992), al parecer por ser áreas libres de sus principales depredadores (Percharde 1970). Los juveniles en cambio frecuentan las praderas de pastos marinos sin formar grandes agregaciones, lo que puede deberse a que se alimentan en las noches permaneciendo enterrados durante el día; además debido a su pequeño tamaño y a la complejidad estructural de las praderas de pastos marinos, es difícil hallarlos en este tipo de hábitat durante las horas luminosas del día (Sanders 1988). Díaz \& Puyana (1994) escriben que en el Caribe colombiano la especie frecuenta fondos de 
arena fangosa con Thalassia y zonas cenagosas de manglar con alta salinidad, encontrándose hasta los $15 \mathrm{~m}$ de profundidad.

S. pugilis fue la especie de gasterópodo más representativo en términos poblacionales de la plataforma continental de La Guajira. Al parecer, la especie es abundante en el mar Caribe y en otras regiones del Atlántico occidental, como también lo demuestran las densidades registradas en distintas regiones del Golfo de México, reportándose densidades mayores a los $2000 \mathrm{ind} . /$ ha (Cervera-Cervera et al. 2007, Canul et al. 2009), semejantes a las encontradas en este estudio.

Dentro de los gasterópodos de mayor tamaño en La Guajira (>120mm LT), T. angulata fue la especie más frecuente en toda el área de estudio. La especie se caracteriza por presentar una amplia distribución en el Caribe y las Antillas (Rodríguez et al. 2003), encontrándose en distintos tipos de hábitats como manglares, ciénagas, áreas rocosas y arrecifes mar afuera, fondos de arena o arena fangosa y en praderas de fanerógamas marinas hasta los $50 \mathrm{~m}$ de profundidad (Vokes \& Vokes 1983, Díaz \& Puyana 1994, McClanahan 2002, Baqueiro-Cárdenas 2004, Aguirre-Aguirre 2006, Cervera-Cervera et al. 2007). En este estudio $T$.angulata se encontró entre los 8-13m de profundidad en todos los tipos de hábitat registrados, siendo más abundante en hábitats compuestos por sustratos de cascajo, gorgonáceos y esponjas, pero con menor afinidad por los hábitats compuestos por pastos marinos. No obstante, la densidad promedio de T. angulata encontrada sobre las praderas de pastos marinos de La Guajira fue mucho mayor a la registrada en años anteriores en el mismo escenario por Aguirre-Aguirre (2006). Sin embargo, debido a las diferencias metodológicas, podríamos solo mencionar que dicha población se ha mantenido en el tiempo en este tipo de hábitat. En cuanto a la relación encontrada por el modelo GAM entre la abundancia de $T$. angulata con el porcentaje de materia orgánica del sedimento, esta reafirma la amplia distribución del caracol en el área de estudio, ya que los valores de $\% \mathrm{MO}$ en donde hubo mayores abundancias
(3-5\%MO) son encontrados en todos los tipos de hábitat registrados.

La amplia distribución y abundancia reflejada en los resultados del índice de valor de importancia sugieren que $T$. angulata presenta una población considerable en la plataforma continental de La Guajira. Con una talla promedio de $178.9 \pm 47.1 \mathrm{~mm}$ y una densidad poblacional aproximada entre los 35-44 ind./ ha, esta especie puede considerarse de interés comercial como parte del recurso caracol en La Guajira. En el estado de Yucatán, Golfo de México, la especie es la segunda más abundante e igualmente la más frecuente, con una longitud promedio de $232 \pm 52 \mathrm{~mm}$ y una densidad aproximada de 4.12 ind./ha (Pérez-Pérez \& Aldana-Aranda 2000). Según esto, la densidad poblacional de $T$. angulata en La Guajira es mayor a la reportada en la península de Yucatán, pero con una talla promedio menor. Esto podría deberse a los controles de pesca que se imponen en esta región de México, donde existe una talla mínima de captura para la especie (Baqueiro-Cárdenas 2004).

En el caso de $V$. muricatum, la especie frecuentó los hábitats estructuralmente más complejos como los compuestos por gorgonáceos, esponjas, cascajo y fondos mixtos. $\mathrm{La}$ especie se distribuye ampliamente en el Caribe y las Antillas, frecuentando fondos rocosos y coralinos, praderas de Thalassia, arenas y cascajo (McClanahan 1992, 2002, Rodríguez et al. 2003). Según Díaz \& Puyana (1994), en el Caribe colombiano la especie se encuentra en fondos de arena en aguas someras hasta $20 \mathrm{~m}$ de profundidad, pero los resultados del presente trabajo demuestran que en La Guajira este es el sustrato menos frecuentado por esa especie. Por otro lado, los datos confirman que V. muricatum es el gasterópodo más abundante en las praderas de pastos marinos de La Guajira, como lo expresó Aguirre-Aguirre (2006).

En el tema poblacional, $V$. muricatum presentó una densidad promedio (51.8 \pm 91.2 ind./ ha) mayor a las reportadas por Pérez-Pérez \& Aldana-Aranda (2000) en el Golfo de México (0.34ind./ha) y por McClanahan (2002) en Belice y La Florida $(1.45 \pm 0.32$ y $0.56 \pm 0.1$ ind./ 
ha, respectivamente). Igualmente, se encontró que las densidades en los hábitats de sustrato duro como el de Gorgonáceos y esponjas sobre cascajo (GEC $=252.5 \pm 104.77$ ind./ha) y Fondo mixto $(\mathrm{FMx}=156.81 \pm 94.26 \mathrm{ind}$./ha) son mucho mayores que las reportadas en la Florida para ambientes de gorgonáceos y algas carnosas ( $1.32 \pm 1.62 \mathrm{ind} . / \mathrm{ha})$ y para Belice en ambientes mixtos de sustrato duro, arena y praderas de pastos (1.45 \pm 0.32 ind./ha, McClanahan 1992, 2002). Esto sugiere que la población de $V$. muricatum en el área de estudio se encuentra en buenas condiciones en comparación con otras regiones del Caribe.

En cuanto a $P$. margaritensis, C. madagascariensis y C. tuberosa, otras especies de gran tamaño, este es el reporte más completo sobre su abundancia y distribución en alguna región del Caribe. Aguirre-Aguirre (2006) registró estas características poblacionales pero únicamente en las praderas de pastos en $\mathrm{La}$ Guajira. P. margaritensis parece preferir los hábitats estructuralmente menos complejos, como extensas áreas de arena y cascajo con algunos elementos vivos dispersos como pastos, algas o corales. En general, la especie tiene una abundancia más o menos apreciable en La Guajira. En cuanto a los ocho ejemplares pertenecientes al género Cassis, cuatro se encontraron en praderas de pastos marinos, pero debido a esta baja abundancia no se puede concluir que sea este su hábitat usual.

La densidad poblacional de S. gigas en la región de La Guajira (tres individuos en 5.7ha evaluadas) ha sido hasta ahora la más baja registrada en Colombia y el mar Caribe (Cuadro 8). Los tres individuos encontrados en este estudio se hallaron en ambientes distintos y estaciones muy separadas (entre 6-240km), indicando que su población posiblemente se encuentra muy dispersa en el área de estudio y muy por debajo del umbral crítico $(<50$ ind./ ha) para una reproducción exitosa (Stoner \& Ray-Culp 2000). Esos autores proponen que por debajo de ese umbral la población podría no tener progenie y el reclutamiento dependería sólo de fuentes externas.
Los hábitats más usados por juveniles y adultos de $S$. gigas son las praderas de pastos marinos (especialmente de T. testudinum), el cascajo arrecifal, los fondos duros cubiertos de macroalgas y las praderas mixtas de pastos y macroalgas (Stoner 2003). Se esperaría entonces que la región de La Guajira, caracterizada por presentar las praderas de pastos y macroalgas más extensas del país (Díaz-Pulido 2000, Gómez-López 2009, Nieto-Bernal et al. 2011), albergara una importante población de caracol pala, lo que es contrario a lo hallado en este estudio. Sin embargo, durante aproximadamente dos décadas, los hábitats someros de la plataforma continental de la Guajira junto con su fauna fueron objeto de una gran presión destructiva a causa de los arrastres camaroneros (Viaña et al. 2002, Duarte et al. 2006, Flórez-Leiva et al. 2007). Esto podría estar asociado con la baja abundancia de $S$. gigas. La variabilidad ambiental de los ecosistemas marinos generados por la acción antrópica a largo plazo, se manifiesta en la variación de la distribución natural de los hábitats, generando cambios en la dinámica poblacional de $S$. gigas (Torres \& Sullivan-Sealey 2002b).

De acuerdo a las observaciones de individuos vivos de las especies de gasterópodos encontradas en la plataforma continental de La Guajira, el grupo de caracoles con abundancias adecuadas para ser de interés comercial está compuesto por S. pugilis, T. angulata, V. muricatum y Phyllonotus margaritensis.

Importancia comercial: Los concheros encontrados en las playas de La Guajira proporcionaron información sobre la composición y prelación de las especies de caracol que son aprovechadas por los pescadores artesanales en la región en los últimos años. Es de aclarar que debido a que los concheros mencionados en este estudio no obedecen a sitios específicos donde los pescadores han frecuentado desechar las conchas a través del tiempo, es imposible generar bajo esta metodología un análisis histórico sobre la pesquería del recurso en la región, como se ha logrado realizar en otras regiones del Caribe (Stoner \& Ray 1996, Stoner 1997, 
CUADRO 8

Densidades de las poblaciones de Strombus gigas en distintas regiones del Caribe

TABLE 8

Densities of Strombus gigas populations in several regions in the Caribbean

\begin{tabular}{|c|c|c|c|}
\hline Localidad & Método & Densidad (ind./ha) & Autor \\
\hline \multicolumn{4}{|l|}{ México } \\
\hline Banco Chinchorro. Q. Roo & áreas circulares & 1555 & Basurto et al. 2007 \\
\hline Banco de Cozumel. Q. Roo & Transectos & 488 & Basurto et al. 2007 \\
\hline Banco Chinchorro. Q. Roo & Transectos & 2500 & Aldana-Aranda et al.2003 \\
\hline Banco de Cozumel. Q. Roo & Transectos & 820 & Aldana-Aranda et al. 2003 \\
\hline Alacranes & Transectos & 84 & Aldana-Aranda et al. 2003 \\
\hline \multicolumn{4}{|l|}{ Estados Unidos } \\
\hline Islas Vírgenes & Transectos & 9.7 & Aldana-Aranda et al. 2003 \\
\hline Puerto Rico & Transectos & 2.6 & Aldana-Aranda et al. 2003 \\
\hline Florida Keys & Transectos & 9.7 & Aldana-Aranda et al. 2003 \\
\hline Belice & - & 88 & Carcomo 2006 \\
\hline Antigua. Bermudas & - & 3.7 & FAO 2007 \\
\hline Honduras & - & 138 & FAO 2007 \\
\hline Nicaragua & - & 230 & FAO 2007 \\
\hline Antillas Holandesas & - & 430 & FAO 2007 \\
\hline \multicolumn{4}{|l|}{ Colombia } \\
\hline Islas del Rosario & áreas circulares & 4 & Gómez et al. 2010 \\
\hline Islas de San Bernardo & áreas circulares & 3.6 & Ballesteros et al. 2005 \\
\hline Banco Serrana. San Andrés & Transectos & 235 & Prada et al. 2008 \\
\hline Banco Roncador. San Andrés & Transectos & 193 & Prada et al. 2008 \\
\hline Banco Quitasueño. San Andrés & Transectos & 49 & Prada et al. 2008 \\
\hline Guajira & Transectos & 0.53 & Presente estudio \\
\hline Umbral crítico de reproducción & & 56 & Stoner \& Ray-Culp 2000 \\
\hline
\end{tabular}

Torres \& Sullivan-Sealey 2002a, Schapira et al. 2009).

El registró de conchas de T. angulata en las playas de La Guajira sugiere que, actualmente, este gasterópodo es el más explotado en la región, con una talla media de pesca de $207 \pm 52.94 \mathrm{~mm}$ LT, cercana a la talla mínima de captura permitida en el Caribe mexicano (205mm, Baqueiro-Cárdenas 2004). Sin embargo; al comparar los datos de densidades de T. angulata de este estudio con los de Aguirre-Aguirre (2006) en los pastos marinos de La Guajira, estos se han mantenimiento en el tiempo; por lo cual podría suponerse que la presión pesquera sobre la especie en la región es relativamente baja o no es constante. Según comentarios de los pescadores de la región, la pesca de T. angulata es incidental, puesto que la mayoría de las veces ésta sale enredada en los "trasmallos" (arte de pesca) que ellos utilizan. Por lo tanto, debería evaluarse la pesquería de este recurso en la región para confirmar esta información.

T. angulata es la especie de gasterópodo más explotada en el Caribe mexicano, aportando aproximadamente el $70 \%$ de la producción total de caracoles del Golfo de México (SantosValencia et al. 2007, 2010). El aprovechamiento de la especie inició en la década de los 80's (Baqueiro 1997), y es actualmente manejado con medidas derivadas del conocimiento de su ciclo reproductivo (Santos-Valencia et al. 2010).

Por otro lado, las conchas de S. gigas encontradas en las playas sugieren la existencia reciente de una población en La Guajira, y la 
posible influencia de la pesca artesanal en su reducción. La evaluación de los concheros en las playas sugiere alguna presión de pesca en el pasado sobre el caracol pala $S$. gigas, pues se encontró un buen número de conchas, donde el $67 \%$ presentaron una longitud total superior a la talla media de madurez propuesta para la región de San Andrés y Providencia (241mm LT, Ávila-Poveda \& Baqueiro-Cárdenas 2006); sin embargo, no existen datos de estadística pesquera que sustenten esta idea.

El estado actual de la población de $S$. gigas en La Guajira podría tener dos explicaciones: la primera, que debido a la explotación en el pasado (visto en los concheros) y teniendo en cuenta su reproducción denso-dependiente, se redujo la posibilidad de recuperación de una población posiblemente aislada, dependiente de procesos de auto-reclutamiento. Y la segun$\mathrm{da}$, es que potencialmente la población de caracol pala de La Guajira nunca fue tan grande como para soportar una explotación continua$\mathrm{da}$, siendo rápidamente disminuida.

El encuentro de conchas de C. madagascariensis y C. tuberosa revela que hay una extracción artesanal de estas especies en La Guajira. Ambas especies están catalogadas como especies vulnerables (VU, A2d) en el Libro rojo de invertebrados marinos de Colombia, según las categorías y criterios dados por la Unión Internacional para la Conservación de la Naturaleza y los Recursos Naturales UICN (2001); en él se proponen como medidas de conservación la realización de un ordenamiento pesquero basado en la evaluación de sus poblaciones y de la presión por pesca (Ardila et al. 2002).

En cuanto a $S$. pugilis, aunque no hubo evidencia de extracción en La Guajira, se sabe que en Colombia se usa su concha para la elaboración de artesanías (Obs. Pers. 2010). Sin embargo se desconoce una extracción dirigida hacía ese recurso. En México, la especie es utilizada como alimento y el recurso fue designado como de importancia comercial, sugiriéndose una talla mínima de captura de $78 \mathrm{~mm}$ LT (Baqueiro-Cárdenas 2004). Esa talla mínima de captura está por encima de la longitud total promedio encontrada en La Guajira
$(68.1 \pm 25.4 \mathrm{~mm})$, donde solo el $6 \%$ de los individuos superan esa talla.

La evaluación de conchas vacías con señales de aprovechamiento en las playas de La Guajira muestra que en los últimos años son cuatro las especies aprovechadas por los pescadores de la región: T. angulata, $S$. gigas, $C$. madagascariensis y C. tuberosa. Estas mismas especies también forman parte de los recursos marinos explotables de México, Honduras y República Dominicana, donde existen medidas de control de pesca con vedas y tallas mínimas de captura para algunas de estas especies (Baqueiro-Cárdenas 2004, FAO 2004-2011).

Como conclusión de este trabajo, se consideran importantes las poblaciones de los caracoles $T$. angulata, S. pugilis y $V$. muricatum, las cuales fueron frecuentes y con amplias distribuciones dentro de la región, además con densidades bien representadas en comparación con otras regiones del Caribe. T. angulata parece ser el caracol más aprovechado en La Guajira, con una presión reflejada en los concheros que se visitaron a lo largo de todo el litoral del área de estudio. No obstante, es importante continuar evaluando todo el recurso para desarrollar medidas de aprovechamiento y conservación. Así mismo, con el fin de concretar la descripción poblacional de $S$. gigas en La Guajira, se recomienda evaluar la conectividad genética de esta población con otras del Caribe colombiano.

\section{AGRADECIMIENTOS}

La presente investigación fue realizada en el marco del proyecto "Distribución y valoración del estado de la población silvestre de caracol pala Strombus gigas (Gasteropoda: Strombidae) en la Guajira (Caribe Colombiano) como guía para su manejo pesquero y conservación", Código 2105-452-21108, Contrato 663 de 2008 de COLCIENCIAS. Agradecemos el apoyo financiero del Instituto de Investigaciones Marinas y Costeras "José Benito Vives de Andréis" INVEMAR, mediante los proyectos BPIN ABM y PNIBM 2009 y BPIN BEM 2010 y a la Secretaría de Pesca y Acuicultura 
del Departamento Archipiélago de San Andrés, Providencia y Santa Catalina. Agradecemos muy especialmente a Eduardo Jaraba por su esencial apoyo logístico en campo. Esta es la contribución N. ${ }^{\circ}$ CTRB-1115 del INVEMAR.

\section{RESUMEN}

En el Caribe continental colombiano el aprovechamiento del recurso caracol y el estado de sus poblaciones han sido poco estudiados, esto se refleja en la carencia de un ordenamiento pesquero. Se evaluó la composición, densidad poblacional y distribución de las especies que conforman ese recurso en La Guajira. Se realizaron censos visuales entre septiembre y noviembre 2009 en 145 transectos de $100 \times 4 \mathrm{~m}\left(400 \mathrm{~m}^{2}\right)$, abarcando un área de $56920 \mathrm{~m}^{2}$ entre Riohacha y el Cabo de la Vela. También se evaluó la composición, abundancia y talla de conchas de gasterópodos en depósitos de conchas vacías en 13 playas. En octubre 2010 se evaluaron 40 transectos más $\left(16000 \mathrm{~m}^{2}\right)$, desde el sur de Riohacha hasta Camarones. Se encontraron 9911 caracoles de 12 especies, siendo Strombus pugilis el más abundante con 8912 individuos y densidad de 1 $538.4 \pm 3662.6$ ind./ha, seguido por Vasum muricatum con 374 individuos y densidad de 51.8 \pm 91.2 ind./ha. El índice de valor de importancia (IVI) de caracoles vivos y de conchas en las playas, muestran a Turbinella angulata como la especie más aprovechada por pescadores artesanales. Los resultados denotan la urgencia de realizar planes de manejo pesquero para este recurso en la región.

Palabras clave: recurso caracol, gasterópodos, Turbinella angulata, La Guajira, Caribe colombiano.

\section{REFERENCIAS}

Aguirre-Aguirre, A. 2006. Comparación estacional de la comunidad de macroinvertebrados epibentónicos asociados a praderas de Thalassia testudinum en La Guajira, Caribe colombiano. Trabajo de grado para optar el título de Biólogo, Pontificia Universidad Javeriana, Bogotá, Colombia.

Aldana-Aranda, D., M. Sánchez-Crespo, V. Patiño-Suárez, A. George-Zamora, E. Baqueiro-Cárdenas, E. Carillo \& S. Pérez. 2003. Abundancia, frecuencia de tallas y distribución espacial del caracol rosa Strombus gigas en el Parque Xel-há, México, p. 47-55. In D. Aldana Aranda (ed.). El Caracol Strombus gigas: Conocimiento Integral para su Manejo Sustentable en el Caribe. CYTED. Programa Iberoamericano de Ciencia y Tecnología para el Desarrollo. Yucatán, México. Special session at the $55^{\text {th }}$ annual meeting Gulf and Caribbean Fisheries Institute.
Appeldoorn, R.S. \& W. Rolke. 1996. Stock abundance and potential yield of Queen Conch resource in Belize. Technical report CARICOM fisheries research assessment and management program (CFRAMP) and the Belize Department of Fisheries, Belize City, Belize.

Ardila, N., G.R. Navas \& J. Reyes. 2002. Libro rojo de invertebrados marinos de Colombia. INVEMAR. Ministerio de Medio Ambiente. La serie Libros rojos de especies amenazadas de Colombia, Bogotá, Colombia.

Ávila-Poveda, O.H. \& E.R. Baqueiro-Cárdenas. 2006. Size at sexual maturity in the queen conch Strombus gigas from Colombia. Bol. Inv. Mar. Cos. 35: 223-233.

Ballesteros, F., C. García, M. Rueda, K. Gómez \& L.S. Mejía. 2005. Relative Abundance and Fishery Characterization of Queen Conch Strombus gigas (Mesogastropoda: Strombidae) in the Archipelago of San Bernardo, Colombian Caribbean. GCFI 58: 393-398.

Baqueiro, C.E. 1997. The Molluscan fisheries of Mexico, p. 39-49. In C.L. MacKenzie, Jr., V.G. Burrell, Jr., A. Rosenfield \& W.L. Hobart (eds.). 1997. The history, present condition, and future of the molluscan fisheries of North and Central America and Europe, Volume 2, Pacific coast and supplemental topics. U.S. Dep. Commer., NOAA Tech. Rep. NMFS 128, 217 p.

Baqueiro-Cárdenas, E.R. 2004. Current state of molluscan resources of the Gulf of Mexico. In M. Caso, I. Pisanty \& E. Ezcurra (eds.). Environmental analysis of the Gulf of Mexico. Harte Research Institute for Gulf of Mexico Studies Special Publication Series No. 1. Secretaría de Medio Ambiente y Recursos Naturales, Instituto Nacional de Ecología (México), Instituto de Ecología, A.C. (México), Harte Research Institute for Gulf of Mexico Studies, Texas A\&M University- Corpus Christi. (También disponible en línea: http://www.harteresearchinstitute.org/images/ Environmental_Analysis/10.pdf).

Basurto, M., P. Cadena, G. Escobedo \& F. Figueroa. 2007. Estimación de biomasa explotable del caracol Rosado Strombus gigas en los bancos abiertos a la pesca: Chinchorro y Cozumel. Foro Regional de Caracol del Golfo de México y Mar Caribe. (Consultado: 29 setiembre 2010, http://www.inapesca.gob.mx/portal/ documentos/publicaciones/MEMORIAS $\% 20 \mathrm{DE} \% 20$ FORO\%201/10.pdf).

Beltrán, T.C.S \& A.B. Villaneda. 2000. Perfil de la pesca y la acuicultura en Colombia. Instituto Nacional de Pesca y Acuicultura-INPA Subdirección de Investigaciones. Bogotá, D.C. Colombia, 26 p.

Berg, C.J. \& D.A. Olsen. 1989. Conservation and management of queen conch (Strombus gigas) fisheries in the Caribbean, p. 421-442. In J.F. Caddy. (ed.). Offprints from marine invertebrate fisheries: Their assessment and management. Wiley, Nueva York, EE.UU. 
Brower, J., J. Zar \& C. Ende. 1998. Field and laboratory methods for general ecology. McGraw-Hill, Nueva York, EE.UU.

Buchanan, J. 1984. Sediment analysis, p. 41-64. In N.A. Holme \& A.D. McIntyre (eds.). Methods for the study of marine benthos. Blackwell, Oxford, Inglaterra.

Canul, A., J.A. Tello-Cetina, H. Álvarez-Hernández \& G. Rivera-Muñoz. 2009. Distribución y abundancia del caracol Strombus pugilis (Linneaeus, 1758) en las costas de Sisal y Celestún, Yucatán, México. Memorias del XIII Congreso Latinoamericano de Ciencias del Mar y VIII Congreso de Ciencias del Mar, COLACMAR, La Habana, Cuba.

Carcomo, 2006. Informe nacional sobre la pesquería del caracol rosa (strombus gigas) en Belize. Belize Fisheries Department Ministry of Agriculture and Fisheries. Belize City, Belize C.A. 15 p.

Catterall, C.P. \& I.R. Poiner. 1983. Age and Sex-dependent Patterns of Aggregation in the Tropical Gastropod Strombus luhuanus. Mar. Biol. 77: 171-182.

Cervera-Cervera, K., C.M. Medina-Martínez, E.F. CobPech \& J.C. Espinoza-Méndez. 2007. Estimaciones de abundancia del recurso caracol para la costa del estado de Campeche, durante las temporadas 20062007. Foro Regional de Caracol del Golfo de México y Mar Caribe (Consultado: 29 septiembre 2010, www.inapesca.gob.mx/portal/documentos/publicaciones/MEMORIAS\%20DE\%20FORO\%201/4.pdf).

Crawley, M.J. 2007. The R book. Wiley \& Sons, Chichester, Reino Unido.

Criales-Hernández, M., C. García \& M. Wolff. 2006. Flujos de biomasa y estructura de un ecosistema de surgencia tropical en La Guajira, Caribe colombiano. Rev. Biol. Trop. 54: 1257-1282.

Díaz, J.M. \& M. Puyana. 1994. Moluscos del Caribe Colombiano: Un catálogo ilustrado. Colciencias, Fundación Natura, INVEMAR, Bogotá, Colombia.

Díaz-Pulido, G. 2000. Vegetación marina de un sector de la plataforma continental de La Guajira (Caribe colombiano). Bol. Invest. Mar. Cost. 29: 27-34.

Duarte, L.O., P Gómez-Canchong, L. Manjarrés, C. García, F.D. Escobar, J. Altamar, J. Viaña, K. Tejada, J. Sánchez \& F. Cuello. 2006. Variabilidad circadiana de la tasa de captura y la estructura de tallas en camarones e ictiofauna acompañante en la pesquería de arrastre del Mar Caribe de Colombia. Invest. Mar. 34: 23-42.

FAO. 2004-2011. Perfiles sobre la pesca y la acuicultura por países. Honduras, República Dominicana. Perfiles sobre la pesca y la acuicultura por países. In Departamento de Pesca y Acuicultura de la FAO, Roma, Italia. (Consultado: 21 noviembre 2011, http:// www.fao.org/fishery/countryprofiles/search/es).

FAO. 2005. Review of the state of world marine fishery resources. FAO Fisheries Technical Paper 457.
Marine Resources Service, Fishery Resources Division, FAO Fisheries Department Food and Agriculture Organization of the United Nations, Roma, Italia.

FAO. 2007. Report of the Regional workshop on the monitoring and management of queen conch, Strombus gigas. Kingston, Jamaica. FAO Fisheries Report No. 832, Roma, Italia.

Flórez-Leiva, L., L. Manjarrés-Martínez, P. GómezCanchong, L.O. Duarte \& G. Díaz-Pulido. 2007. Macroalgas marinas afectadas por la flota de arrastre camaronero en el Mar Caribe de Colombia. Rev. Acad. Colomb. Cienc. 31: 41-48.

Folk, R.L. 1980. Petrology of sedimentary rocks. Hemphill, Austin, Texas, EE.UU.

Gómez-Campo, K., M. Rueda \& C. García-Valencia. 2010. Distribución espacial, abundancia y relación con características del hábitat del caracol pala Eustrombus gigas (Linnaeus) (Mollusca: Strombidae) en el Archipiélago Nuestra Señora del Rosario, Caribe colombiano. Bol. Invest. Mar. Cost. 39: 137-159.

Gómez-López, D.I. 2009. Estado de las praderas de pastos marinos. In INVEMAR 2009. Informe del estado de los ambientes y recursos marinos y costeros en Colombia: Año 2008. Serie de publicaciones periódicas No. 8. Santa Marta. Colombia.

Hastie, T.J. \& R.J. Tibshirani. 1990. Generalized additive models. Chapman and Hall. Londres, Reino Unido.

Kitutani, K. \& H. Yamakawa. 1999. Marine snails seed production towards restocking enhancement basic manual. Field Document No. 14. Tonga Aquaculture Research \& Development Project, Japan International Cooperation Agency. South Pacific Aquaculture Development Project (II), Food and Agriculture Organization of the United Nations.

Lamprecht, H. 1990. Silvicultura en los Trópicos; Los ecosistemas Forestales en los bosques Tropicales y sus especies arbóreas posibilidades y métodos para un aprovechamiento sostenido. Traducción del alemán de Antonio Carrillo. Deutsche Gesellschaftfur. Technische Zusammenarbeit (GTZ) Gmobh. Rep. Federal de Alemania.

Leal, J.H. 2002. Gastropods. In K.E Carpenter. (ed.). The living marine resources of the Western Central Atlantic. Volume 1. Introduction, molluscs, crustaceans, hagfishes, sharks, batoid fishes and chimaeras. FAO species identification guide for fishery purposes, American Society of Ichthyologists and Herpetologists and the European Commission. Food and Agriculture Organization of the United Nations, Roma, Italia.

Luczak, C., M. Janquin \& A. Kupka. 1997. Simple standard procedure for the routine determination of organic matter in marine sediment. Hydrobiology 345: 87-94. 
MacIntosh, R.A. 1980. The snail resource of the Eastern Bering Sea and its fishery. Mar. Fish. Rev. 42: 15-20.

Maguire, J.J., M. Sissenwine, J. Csirke, R. Grainger \& S. Garcia. 2006. The state of world highly migratory, straddling and other high seas fishery resources and associated species. FAO Fisheries Technical Paper 495. Food and Agriculture Organization of the United Nations, Rome, Italy.

McClanahan, T.R. 1992. Epibenthic gastropods of the Middle Florida Keys: the role of habitat and environmental stress on assemblage composition. J. Exp. Mar. Biol. Ecol. 160: 169-190.

McClanahan, T.R. 2002. A comparison of the ecology of shallow subtidal gastropods between western Indian Ocean and Caribbean coral reefs. Coral Reefs 21: 399-406.

Nash, W.J. 1993. Trochus, p. 451-497. In A. Wright \& L. Hill (eds). Nearshore Marine Resources of the South Pacific. Institute of Pacific Studies-Suva, Forum Fisheries Agency-Honiara, International Centre for Ocean Development-Canada.

Nieto-Bernal, R., A. Rodríguez, L. Chasqui, E. Castro \& D.L. Gil-Agudelo. 2011. Distribución y abundancia de las poblaciones de gasterópodos de importancia comercial en La Guajira, Caribe colombiano. Instituto de Investigaciones Marinas y Costeras -INVEMAR, Subsecretaría de Pesca de la Gobernación de San Andrés, Providencia y Santa Catalina. Serie de Documentos Generales de INVEMAR No. 46. Santa Marta, Colombia.

Osorno, A., D.L. Gil-Agudelo \& L.A. Gómez-Lemos. 2009. Plan de Investigación para la Conservación de Cittarium pica (Linnaeus, 1758). INVEMAR, Serie de Publicaciones Especiales No. 16 Santa Marta, Colombia.

Percharde, P.L. 1970. Further underwater observations on the molluscan genus Strombus (Linneaus) as Found in the Waters of Trinidad and Tobago. Carib. J. Sci. 10: $73-81$.

Percharde, P.L. 1982. A Comparison of the Strombus (Mollusca) Colonies, of two Southern Caribbean Islands: Trinidad and Grenada. Carib. J. Sci. 18: 35-39.

Peréz-Peréz, M. \& D. Aldana-Aranda. 2000. Distribución, abundancia y morfometría de Strombus costatus, Turbinella angulata, Busycon contrarium y Pleuroploca gigantea (Mesogasteropoda: Strombidae, Turbinellidae, Neptuneidae y Fasciolaridae) en Yucatán, México. Rev. Biol. Trop. 48: 145-152.

Poutiers, J.M. \& R. Cipriani. 1992. Gastrópodos y Bivalvos, p. 29-76. In F. Cervigón, R. Cipriani, W. Fischer, L. Garibaldi, M. Hendrickx, A.J. Lemus, R. Márquez, J.M. Poutiers, G. Robaina \& B. Rodríguez (eds.). Fichas FAO de identificación de especies para los fines de la pesca. Guía de campo de las especies comerciales marinas y de aguas salobres de la costa septentrional de Sur América. Roma, Italia.

Prada, M., E. Castro, E. Taylor, V. Puentes, R. Appeldoorn \& N. Daves. 2008. Non-Detrimental findings for the Queen conch (Strombus gigas) in Colombia. NonDetriment Findings Workshop Case Studies, Working Group 9 Aquatic Invertebrates (WG9), Case Study 3, Strombus gigas. Country Colombia. In International Expert Workshop on CITES Non-Detriment Findings Cancún, México, November $17^{\text {th }}$ to $22^{\text {nd }}, 2008$ (Consultado: 29 septiembre 2010, www.conabio.gob.mx/ institucion/cooperacion_internacional/TallerNDF/ Links-Documentos/WG-CS/WG9-AquaticInvertebrates/WG9-CS4\%20Queensland/WG9-CS4.pdf).

Reed, S.E. 1992. Reproductive anatomy, biology and behavior of the genus Strombus in The Caribbean with emphasis on Strombus pugilis. Tesis de Doctorado, Universidad de Puerto Rico, Mayagüez, Puerto Rico.

Robertson, R. 2003. The edible West Indian Whelk Cittarium pica (Gastropoda: Trochidae): natural history with new observations. Proc. Acad. Nat. Sci. Philad. 153: $27-47$.

Rodríguez, L.S., R. Vargas \& J. Cortés. 2003. Biodiversidad marina de Costa Rica: Gastrópodos (Mollusca: Gastropoda) de la costa Caribe. Rev. Biol. Trop. 51: 305-399.

Sanders, I.M. 1988. Energy relations in a population of Strombus pugilis. Tesis de Doctorado, Universidad de Puerto Rico, Mayagüez, Puerto Rico.

Santos-Valencia, J., J. Seca-Escalante, M. Medina-Martínez, D. Murillo-Guerrero \& M. Huchín-Maturel. 2007. La pesquería de caracol en la zona norte de Campeche. Foro Regional de Caracol del Golfo de México y Mar Caribe. (Consultado: 29 septiembre 2010,www.inapesca.gob.mx/portal/documentos/ publicaciones/MEMORIAS\%20DE $\% 20 \mathrm{FORO} \% 20$ 1/3.pdf).

Santos-Valencia, J., I. Martínez, M. Enríquez-Díaz \& A. Dalila-Aldana. 2010. Ciclo Reproductor de Turbinella angulata (Mollusca:Gastropoda) en Campeche, Golfo de México. GCFI 62: 408-414.

Schapira, D., I.A. Montaño, A. Antczak \& J.M. Posada. 2009. Using shell middens to assess effects of fishing on queen conch (Strombus gigas) populations in Los Roques Archipelago National Park, Venezuela. Mar. Biol. 156: 787-795.

Schmidt, S., M. Wolff \& J.A. Vargas. 2002. Population ecology and fishery of Cittarium pica (Gastropoda: Trochidae) on the Caribbean coast of Costa Rica. Rev. Biol. Trop. 50: 1079-1090.

Schweizer, D. \& J.M. Posada. 2006. Distribution, density and abundance of the Queen conch, Strombus gigas, in Los Roques Archipelago National Park, Venezuela. Bull. Mar. Sci. 79: 243-258. 
Stoner, A.W. 1997. Shell middens as indicators of longterm distributional pattern in Strombus gigas, a heavily exploited marine gastropod. Bull. Mar. Sci. 61: 559-570.

Stoner, A.W. 2003. What constitutes essential nursery habitat for a marine species? A case study of habitat form and function for queen conch. Mar. Ecol. Prog. Ser. 257: 275-289.

Stoner, A.W. \& M. Ray. 1996. Shell remains provide clues to historical distribution and abundance patterns in a large seagrass-associated gastropod (Strombus gigas). Mar. Ecol. Prog. Ser. 135: 101-108.

Stoner, A.W. \& M. Ray-Culp. 2000. Evidence for Allee Effects in an Over-Harvested Marine Gastropod: Density-Dependent Mating and Egg Production. Mar. Ecol. Prog. Ser. 202: 297-302.

Stotz, W. 1997. Las áreas de manejo en la ley de pesca y acuicultura: Primeras experiencias evaluación de la utilidad de esta herramienta para el recurso Loco. The management areas in the fishery law: First experiences and evaluation of utility as a management tool for Concholepas concholepas. Estud. Oceanol. 16: $67-86$.

Suzunaga, J., L. Calero \& A. Molina. 1992. Síntesis del estudio de las condiciones oceanográficas al N-E de La Guajira. Fase II. Bol. Cient. C.I.O.H. 11: 59-69.

Theile, S. 2002. "Queen conch and the Review of Significant Trade". CITES World Official Newsletter of the Parties 10: 3-4.
Torres, R.E. \& K.M. Sullivan-Sealey. 2002a. Shell midden surveys as source of information about fished Queen conch (Strombus gigas) populations: A case study in Parque Nacional del Este, Dominican Republic. GCFI 53: 143-153.

Torres, R.E. \& K.M. Sullivan-Sealey. 2002b. Abundance, size frequency, and spatial distribution in Southeastern Dominican Republic: A four-year population study in Parque National del Este. GCFI 53: 120-128.

UICN. 2001. Categorías y Criterios de la Lista Roja de la UICN: Versión 3.1. Comisión de Supervivencia de Especies de la UICN. UICN, Gland, Suiza y Cambridge, Reino Unido.

UNEP-WCMC. 2011. UNEP-WCMC Species Database: CITES-Listed Species (Consultado: 11 Octubre, 2012, http://www.unep-wcmc-apps.org/isdb/CITES/ Taxonomy/tax-genus-result.cfm/isdb/CITES/Taxonomy/tax-genus-result.cfm?displaylanguage $=$ esp $\&$ Genus $=4618 \&$ source $=$ animals $\&$ Country $=$ ).

Viaña, J., Medina, A., Manjarres \& L.M. Barros. 2002. Evaluación de la ictiofauna demersal extraída por la pesquería industrial de arrastre en el área norte del Caribe colombiano (enero/2000-junio/2001). En: Informe Técnico Final del Proyecto Evaluación de las pesquerías demersales del área norte del Caribe colombiano y parámetros ecológicos, biológico-pesqueros y poblacionales del recurso Pargo (INPACOLCIENCIAS), Santa Marta.

Vokes, H.E. \& E.H. Vokes 1983. Distribution of shallowwater marine mollusca, Yucatan Peninsula, Mexico. Middle American Research Institute 54: 1-183. 